\title{
EFFECT OF MEDICATION RELATED EDUCATIONAL INTERVENTIONS ON IMPROVING MEDICATION ADHERENCE IN PATIENTS WITH TYPE 2 DIABETES MELLITUS
}

\author{
NIMMY ELIZABETH GEORGE*, SMRITHY SUNNY, ANITTA MARIYAM SAM, ALEENA SUSAN SABU, \\ PRUDENCE A RODRIGUES
}

Department of Pharmacy Practice, PSG College of Pharmacy, Coimbatore, Tamil Nadu, India. Email: nimgeorge2@gmail.com

Received: 26 August 2017, Revised and Accepted: 07 October 2017

\begin{abstract}
Objectives: To assess the patient medication adherence using 8 item morisky medication adherence scale (MMAS). To categorize patients based on their adherence to antidiabetic medications into low, medium, and high adherent. To provide educational interventions such as patient counseling using teach-back method, patient medication information leaflet, and audio-visual aids and thus to improve the patient medication adherence.

Methods: After obtaining informed consent from the patients, data of the patients were recorded in data collection forms. Their adherence to antidiabetic medication was evaluated using 8 item MMAS and patients were categorized into low, medium, and high adherence groups based on the score. Counseling was done based on the categorization (high, medium, and low). During review, again adherence was rechecked using 8 item MMAS.

Results: Medication adherence was measured using 8 item MMAS on review and adherence was found to be improved using different patient counseling methods according to their adherence category. Improvement in score within low adherence group was found to be $83.87 \%$; improvement of the low adherence group to medium adherence group was $16.12 \%$. Improvement within the medium adherence group was $82.14 \%$ and from medium adherence to high adherence group was $17.85 \%$.
\end{abstract}

Conclusion: Patient counseling can improve adherence in type 2 diabetes mellitus patients, which in turn help patients in achieving optimal glycemic control.

Keywords: Medication adherence, Morisky medication adherence scale, Diabetes mellitus.

(c) 2018 The Authors. Published by Innovare Academic Sciences Pvt Ltd. This is an open access article under the CC BY license (http://creativecommons. org/licenses/by/4. 0/) DOI: http://dx.doi.org/10.22159/ajpcr.2018.v11i1.22238

\section{INTRODUCTION}

Diabetes mellitus, an emerging global epidemic metabolic disorder characterized by increased blood glucose levels or hyperglycemia $[1,2]$. It is associated with abnormalities in carbohydrate, fat, and protein metabolism. The complications of diabetes mellitus include microvascular and macrovascular disorders as well as neuropathic manifestations [3]. Over the past two decades, there has been a rapid increase in the worldwide prevalence of diabetes mellitus and the number of individuals will continue to increase [4]. India is designated as the diabetes capital of the world and is the leading country with the largest number of diabetes subjects [5].

Optimal glycemic control can be achieved by proper diet, lifestyle and the main factor being a strict adherence to medication which in turn reduces long-term complications of diabetes mellitus [6]. Medication non-adherence in patients with type 2 diabetes mellitus has lead to reduced effectiveness of the therapy [7]. The purpose of the study is to improve medication adherence to oral hypoglycemic agents (OHA) and insulin in diabetes patients by first measuring the medication adherence of individual patients using 8 item morisky medication adherence scale (MMAS) and then providing the patient educational interventions such as patient counseling using teach-back method, audio-visual aids (videos), and medication information leaflets based on the score in 8 item MMAS [8].

Patient counseling involves educating patients and their representatives about medication-related information such as direction of use, possible side effects, and precautions of use, diet, and lifestyle modifications orally or in written form. Proper education regarding all the abovementioned aspects of medication plays a significant role in improving medication adherence, which in turn helps in achieving optimal glycemic control [9]. Standard patient counseling may be less effective in this, so combination of multiple patient counseling techniques like to teach-back method, use of patient information leaflets and video results in a better improvement in adherence [10].

Teach-back method is an efficient patient counseling method which not only provides counseling to the patient but also assesses patients' understanding of provided information [11]. It involves initially providing all necessary information regarding drug to the patient, including indication, side effects, precautions, and frequency of the drug, after providing this information, the patient will be asked openended questions such as "when to take this drug," "for what this drug is given," "how to take the drug," "what should you avoid while taking the drug," and "how often you should take the medicine." Teach-back method helps us to ensure that patient has understood the information given and helps to correct misunderstandings if any [12]. Teach-back method also improves memory retention by patients who take new medications.

The videos are quite effective than traditional methods of patient education in improving short-term knowledge of the patients [13]. The method of educating the patients with videos is quite practical and the cost of which is negligible [14]. Videotapes can be produced relatively cheaply and thus helps it to be tailor-made for a given patient population [15].

Patient information leaflets are written information which is produced in simple language about the patient's illness and treatment, including information about medications [16]. It includes information such as name of the medication, generic name, strength, when it has to be taken, how it has to be taken, the main side effects, 
how to overcome side effects, contraindication and precaution [17]. Printed materials reinforce verbal advice and results in improving understanding about the medication information [18-21]. The rationale of the study is to investigate if patient education based on individual score of adherence would help in improving the medication adherence to antidiabetic medications in patients with type 2 diabetes mellitus.

\section{METHODS}

\section{Phase one}

\section{Preparation for the study}

Patient data collection forms, patient medication information leaflet for commonly prescribed antidiabetic drugs and audio-visual aids demonstrating signs and symptoms of hypoglycemia and insulin administration were prepared.

\section{Institutional ethics committee approval}

The ethical clearance for the study was obtained from the Institutional Human Ethics Committee of the PSG Institute of Medical Science and Research.

\section{Phase two}

\section{Patients selection}

Patients were segregated based on inclusion and exclusion criteria.

Inclusion criteria:

- Patients of age 18-70 years of either sex with type 2 diabetes mellitus

- Patients on OHA or insulin.

Exclusion criteria:

- Patients with visual, cognitive, and hearing impairment

- Pregnant and lactating women

- Terminally ill patients

- Patients $<18$ years or $>70$ years.

\section{Obtaining informed consent}

Informed consent form was obtained from the selected patients and English or Tamil.

\section{Patient categorization}

Patients were categorized into highly adherent, medium adherent and low adherent based on 8 item MMAS and it was reported and recorded.

\section{Providing intervention}

High adherent patients were provided with teach-back method patient counseling. Medium adherent patients were provided with teach-back method patient counseling and patient medication information leaflet. Low adherent patients were provided with audio-visual aids, teachback method patient counseling and patient medication information leaflet.

\section{Phase three}

Patient review

After 2 months, patients came for review and their medication adherence was checked again using 8 item MMAS and was reported and recorded.

\section{Statistical analysis}

The gathered data were statistically analyzed based on paired t-test using SPSS software.

\section{RESULTS}

Out of 120 patients selected and included in the study based on inclusion and exclusion criteria 98 patients (82\%) came for review and 22 patients $(18 \%)$ did not turn up for review.
Out of the 98 subjects who completed the study, 61 (62\%) were male and 37 (38\%) were female. Male subjects were predominant compared to female subjects.

In this study, the subjects of age 18-70 years were included. As per the results, maximum subjects $(35.7 \%)$ were in the age group of $50-59$ years, followed by $40-49$ years $(31.6 \%)$. The least number of subjects was from the age of 20-29 years (2.04\%).

Out of the total 98 subjects, most of the subjects were not having any specific social habit (35.71\%). 16 subjects $(16.32 \%)$ were found to be both alcoholic and smoker, 15 (15.30\%) were found to be smokers, and $10.20 \%$ of subjects were alcoholic.

Hypertension was found to be the main comorbid condition associated with diabetes mellitus (43\%) followed by coronary artery disease $(15 \%)$ and dyslipidemia accounting for $13 \%$. Other comorbidities found among diabetes mellitus patients under study were less likely associated with hyperglycemia condition.

Based on 8 item MMAS the patients were classified into high, medium, and low adherence groups. In this study, number of subjects were found to be in low adherence group accounting for $63.2 \%$ and $53.06 \%$ before and after the counseling, respectively, followed by medium $(28.5 \%$ before and $33.6 \%$ after the counseling). The least number of subjects was found to be in a high adherence group ( $8.3 \%$ before and $13.2 \%$ after counseling).

In this study, number of patients who did not turn up for review were from a low adherence group (13 patients) followed by medium adherence group ( 6 patients). Least number of patients who did not turn up for review was from high adherence group.

The patients received both insulin and non-insulin therapy. 62 patients $(63.26 \%)$ were on OHA, 26 patients $(26.53 \%)$ were on both insulin and OHA. 10 patients $(10.20 \%)$ received only insulin.

Out of the 98 subjects enrolled in the study 39 patients (40\%) received monotherapy, i.e., either insulin or a single OHA whereas 59 subjects $(60 \%)$ received more than one drug, i.e., either multiple OHAs or both insulin and OHA.

From the study, it was observed that the most commonly prescribed drug was metformin $(28 \%)$ followed by insulin $(20 \%)$ and vildagliptin plus metformin (14\%). Other drugs prescribed were glimepiride $(10 \%)$, gliclazide $(8 \%)$, gliclazide plus metformin $(10 \%)$, repaglinide (2\%), and other drugs (8\%).

From this study, the main reason among patients for non-adherence was found to be forgetting to take medicines (31\%). $27 \%$ of the patients found it difficult to adhere to medicine while traveling. $24 \%$ of the patients stopped taking medicine when they felt better, and $18 \%$ of the patients stopped medicine as they felt worse after taking the medicine.

Out of the 98 patients involved, 40\% (39 subjects) received monotherapy, and $60 \%$ ( 59 subjects) received two or more drugs. There was a significant positive correlation at 0.01 level ( 2 tailed) between number of drugs and adherence.

From the study, it was observed that there was a significant negative correlation at the 0.01 level ( 2 tailed) between age of the patient and adherence. As age increased, there was a simultaneous decrease in adherence.

All the patients who were categorized in the high adherence group (i.e. 8 subjects before counseling) based on 8 item MMAS before counseling did not show any change in the score after counseling that is they continued to be in high adherence group (i.e. 8 subjects after counseling). 
Patients with medium adherence before providing counseling (i.e., teach-back method and patient information leaflet) showed a significant change in score after counseling $(\mathrm{p}<0.05)$. Some patients continued to be in the medium adherence group (23 subjects) whereas some patients showed a change in the group that is from medium to high (5 subjects) (Table 1$)$.

Patients with low adherence before providing counseling (i.e., teachback method and patient information leaflet, and audio-visual aids) showed a significant change in score after counseling $(p<0.05)$. Some patients showed improvement in score within the low adherence group (52 subjects) whereas some patients showed a change in the group that is from low to medium (10 subjects) (Table 2 ).

\section{DISCUSSION}

Our study was conducted in type 2 diabetes mellitus patients in a tertiary care hospital. Males predominated in this study which is an agreement with the result of the study conducted by Vengurlekar et al. [20]. This may be due to smoking, alcoholic habits, and other lifestyle changes which are seen more commonly in males and these factors have a hallucinating effect on glycemic control.

Majority of the patients belong to the age group of 50-59 (35.7\%), and this can be correlated with the data generated by the center for disease control and prevention, America that the majority of diabetes mellitus patients belong to the age group 50-59 [21]

Hypertension accounted for $43 \%$ of the total comorbidities which is higher than that found in a study conducted by Manjusha et al. which stated that hypertension accounted for only $27.27 \%$ of the total complications [1]. Our study findings are also similar to the study conducted in Texas that hypertension is more common complication affecting $20-60 \%$ of people with diabetes [22].

The study population was divided into patients receiving insulin, OHA and both insulin and OHA. Patients who were on OHA were found to be more non-adherent than patients on insulin and patients receiving both insulin and OHA in our study. This is in contrast to the study conducted by Sajith et al. [1]. In which subjects were more adherent to OHAs. The non-adherence to OHA observed in our study may be due to lack of knowledge about drug therapy.

Adherence to appointment keeping was found to be $82 \%$ in our study which was more than subjects who were non-adherent to appointment keeping (18\%) due to forgetfulness, travel, long clinic wait time, no response and busy schedule of work. This is similar to the result obtained from a study by Sajith $e$ t al. [1] where $98.10 \%$ of subjects were found to be adherent and just $1.90 \%$ of subjects were found to be nonadherent to appointment keeping.

As it is a self-reported method the 8 item MMAS used to assess medication adherence might be associated with some shortcomings; however, there is no gold standard method for assessing adherence. The assessment of patient's response to 8 item MMAS showed that $8.3 \%$ had high adherence, $28.5 \%$ had medium adherence, and $63.2 \%$ had low adherence. This is not in accordance with the study conducted in Pune which showed that $40.95 \%$ had high adherence, $37.14 \%$ had medium, and $21.9 \%$ had low adherence [1]

After providing appropriate educational interventions (teach-back method, patient information leaflet, and audio-visual aids) based on the adherence class, there was a considerable improvement in adherence to $13.2 \%$ of subjects in high adherence, $33.67 \%$ of subjects in medium, and $53.06 \%$ in low adherence groups. This highlights the fact that patient counseling can improve medication adherence as suggested by Carollo et al. [9].

Based on our study forgetfulness (31\%) was the main patientrelated factors that contributed to low adherence in subjects. This is
Table 1: Improvement observed in medium adherence group

\begin{tabular}{lll}
\hline S. No. & \#Changes observed & $\begin{array}{l}* \text { Number of } \\
\text { patients (\%) }\end{array}$ \\
\hline 1 & $\begin{array}{l}\text { Improvement in score within medium } \\
\text { adherence group }\end{array}$ & $23(82.14)$ \\
2 & $\begin{array}{l}\text { Improvement from medium to high } \\
\text { adherence group }\end{array}$ & $5(17.85)$ \\
\hline
\end{tabular}

"Based on score improvement in MMAS, * $(\mathrm{n}=120)$. MMAS: Morisky medication adherence scale

Table 2: Improvement observed in low adherence group

\begin{tabular}{lll}
\hline S. No. & \#Change observed & $\begin{array}{l}\text { *Number of } \\
\text { patients (\%) }\end{array}$ \\
\hline 1 & Improvement within low adherence group & $52(83.87)$ \\
2 & $\begin{array}{l}\text { Improvement from low to medium } \\
\text { adherence group }\end{array}$ & $10(16.12)$ \\
&
\end{tabular}

"Based on score improvement in MMAS, * $(n=120)$. MMAS: Morisky medication adherence scale

in accordance with the study conducted by Manjusha et al. [1]where forgetfulness contributed to $(81.9 \%)$ other patient-related factors that contribute to low adherence are travel (27\%), felt worse (18\%), and felt better $(24 \%)$

Various factors affect adherence in type 2 diabetes mellitus patients. Correlation between various factors and adherence were done in the study. It was observed that age of the patient was positively correlated with adherence, i.e., as age increased there was an increase in adherence rate among patients. This may be due to increased knowledge about the disease and its complications in elderly patients compared to young patients. Similar relation between age of the patients and adherence was observed in another study [14].

However, sex and social habits were not consistently associated with the level of patient adherence [23]. Number of drugs prescribed was positively correlated to adherence, i.e. patients who received monotherapy were more adherent to their medications compared to patients who were on treatment with multiple antidiabetic agents (insulin and OHAs). Similar results were obtained in another study [19]. Diabetic patients may receive antidiabetic drugs as well as drugs for their comorbidities. Thus, drug regimen for diabetic patients will be complex and will affect adherence level of the patients.

\section{CONCLUSION}

It is concluded that the participants in the area of study were less adherent to their antidiabetic medications. Various factors of medication non adherence were re-identified and evaluated. Various educational interventions were provided to the patients according to the adherent scales which included teach-back method patient counseling for high adherence patients, medication information leaflets and counseling for medium adherence, audio-visual aids, patient information leaflets and counseling for low adherence. This study points out that the involvement of clinical pharmacist can play a major role in improving medication adherence in patients with type 2 diabetes mellitus.

\section{REFERENCES}

1. Sajith M, Pankaj M, Pawar A, Modi A, Sumariya R. Medication adherence to antidiabetic therapy in patients with Type 2 diabetes mellitus. Int J Pharm Pharm Sci 2014;6:564-70.

2. Asmini P. The influence of education of drug information by pharmacist on the improvement of knowledge and therapeutic target to Type 2 diabetes mellitus outpatients in hospital. Int J Pharm Pharm Sci 2017:9:199-204.

3. Ahmad NS, Ramli A, Paraidathathu T. Medication adherence in patients with Type 2 diabetes mellitus treated at primary health clinics in Malaysia. Patient Prefer Adherence 2012;6:613-22. 
4. Carlisle A, Jacobson KL, Di Francesco L, Parker RM. Practical strategies to improve communication with patients. Health Lit Pharm 2011;36:576-9.

5. Sakthonng P, Chabunthom R, Charoenvisuthiwongs R. Psychometric properties of Thai version of 8 item Morisky medication adherence scale in patients with Type 2 diabetes mellitus. Ann Pharmacother 2009;43:950-5.

6. Bezie Y, Hernandez NM, Batista R, Niang S, Huet D. Therapeutic compliance: A prospective analysis of various factors involved in the adherence rate in Type 2 diabetes. Diabetes Metab 2006;3:611-6.

7. Omar MS, San KL. Diabetes knowledge and medication adherence among geriatric patient with Type 2 diabetes mellitus. Int J Pharm Pharm Sci 2014;6:103-6.

8. Palaian S, Chhetri A, Prabhu M, Rajan S, Shankar P. Role of pharmacist in counseling diabetes mellitus patients. Internet $\mathrm{J}$ Pharmacol 2004; 4:2-12.

9. Carollo A, Adamo A, Di Giorgio C. The importance of clinical pharmacist counseling in improving patient medication adherence. Eur J Hosp Pharm 2013;52:151-6.

10. Fisher Rowley PT, Lipkin M. Genetic counseling for Beta thalassemia trait following health screening in health maintenance organization: Comparison of programmed and conventional counselling. Am J Hum Genet 1981;33:987-94.

11. Costa E, Giardini A, Savin M, Menditto E, Lehane E, Laosa O, et al. Interventional tools to improve medication adherence: Review of literature. Patient Prefer Adherence 2015;9:1303-14.

12. Lassen LC. Patient compliance in general practice. Scand J Prim Health Care 1989;7:179-80

13. Uzark K, Klos D, Davis W, Rosenthal A. Use of videotape in the preparation of children for cardiac catheterization. Pediatr Cardiol 1982;3:287-91.

14. Jimmy B, Jose J. Patient medication adherence: Measures in daily practice. Oman Med J 2011;3:155-9.

15. Gangliano ME. A literature review on the efficacy of video in patient education. J Med Educ 1988;63:786-91.

16. Jin J, Sklar GE, Min Sen Oh V, Chuen Li S. Factors affecting therapeutic compliance: A review from the patient's perspective. Ther Clin Risk Manag 2008;4:269-86.

17. Zullig LL, Gellard WF, Bosworth HB. Improving diabetes medication adherence: Successful scalable interventions. Patient Prefer Adherence 2015;9:139-49.

18. Sudore RL, Schillinger D. Interventions to improve care for patients with limited health literacy. J Clin Outcomes Manage 2009;16:20-7.

19. Mohammed S, Poudel S, Laloo F, Madhur A, Robert R, Mathew B. Assessment of drug related problems in a tertiary care teaching hospital, India. Asian J Pharm Clin Res 2017;10:310-3.

20. Sivadasan S, Sellapan M. A study on the awareness and attitude towards pharmacovigilance and adverse drug reaction reporting among nursing students in a private university, Malaysia. Int J Curr Pharm Res 2015;7:84-9.

21. Maurya H, Kumar T. A review on comprehensive overview in the management of nephritic disorders. J Crit Rev 2016;3:34-43.

22. Vengurlekar S, Shukla P, Patidar P, Bafna R, Jain S. Prescribing pattern of anti diabetic drugs in Indore city hospital. Indian J Pharm Sci 2008:70:637-40.

23. Center for Disease Control and Prevention. Distribution of Age at Diagnosis of Diabetes Among Adult Incident Cases Aged 18-79 Years. United States: Diseases Public Health Resource; 2011. 